\title{
Analysis and Optimization of Intensity Noise Reduction in Spectrum-Sliced WDM Systems Using a Saturated Semiconductor Optical Amplifier
}

\author{
Mingshan Zhao, Geert Morthier, Senior Member, IEEE, and Roel Baets, Senior Member, IEEE
}

\begin{abstract}
Theoretical and experimental results on the reduction of the intensity noise in spectrum-sliced wavelength-divisionmultiplexing systems using a saturated semiconductor optical amplifier (SOA) are presented. The influence of the injected current and the input power to the saturated SOA on the noise reduction and its bandwidth are studied. The optimum condition for a high noise suppression ratio and a large bandwidth is derived. For the optimum operation of the SOA, an increase of $13.5 \mathrm{~dB}$ in the intensity-noise-limited signal-to-noise ratio for a bitrate of $2.488 \mathrm{~Gb} / \mathrm{s}$ and of $17.5 \mathrm{~dB}$ for a bitrate of $622 \mathrm{Mb} / \mathrm{s}$ are obtained experimentally in a single-stage SOA.
\end{abstract}

Index Terms-Optical fiber communication, optical noise, semiconductor optical amplifier, wavelength-division multiplexing.

\section{INTRODUCTION}

$\mathbf{I}$ NTENSITY noise in spectrum-sliced wavelength-division-multiplexing (WDM) systems utilizing incoherent broad-band sources imposes strong limits on the achievable system performance [1]. Techniques for reducing the intensity noise using a saturated semiconductor optical amplifier (SOA) have been recently proposed [2] and experimentally demonstrated [3], [4]. Such techniques have the advantage of simplicity, high efficiency, and potentially low cost since the saturated SOA can be used simultaneously for both noise reduction and signal modulation [4]. Therefore, this approach is very practical and useful for application in cost-sensitive local area networks. However, previous reports on the noise reduction considered only the dependence of the noise reduction on the gain compression (input power level) of the SOA or consisted of a simple experimental demonstration. In this letter, we present a more complete analysis of the noise reduction in spectrum-sliced WDM systems. The influence of the injected current and the input power level of the SOA on the noise reduction and its bandwidth is studied theoretically and experimentally, and the optimum condition for a high noise suppression ratio and a large bandwidth is derived.

\section{ANALYTICAL THEORY}

We consider a traveling-wave SOA. An optical beam is incident and propagates through the SOA. Neglecting the contribu-

Manuscript received May 30, 2001; revised November 20, 2001. This work was supported by the Flemish Government-IWT.

The authors are with the Department of Information Technology, Ghent University-IMEC, B-9000 Gent, Belgium (e-mail: mszhao@intec.rug.ac.be; morthier@intec.rug.ac.be; baets@ intec.rug.ac.be).

Publisher Item Identifier S 1041-1135(02)01212-0. tion of the spontaneous emission from the SOA itself, the propagation of optical power $P$, and the carrier density $N(z, t)$ in the amplifier can be described by the following equations [5]:

$$
\begin{aligned}
\frac{\partial P}{\partial z}-\frac{1}{v_{g}} \frac{\partial P}{\partial t} & =\left[\Gamma g(N)-\alpha_{\text {int }}\right] P \\
\frac{\partial N(z, t)}{\partial t} & =\frac{J}{q d}-\frac{N(z, t)}{\tau_{d}}-\frac{\Gamma g(N) P}{h \nu A}
\end{aligned}
$$

where $\Gamma$ is the mode confinement, $g(N)$ the gain coefficient, $\alpha_{\text {int }}$ the internal loss, $J$ the injected current density, $d$ the active layer thickness of the SOA, $A$ the active region area, $q$ the electronic charge and $\tau_{d}$ the carrier lifetime.

Now, we consider the case where there is a large saturating power of the optical beam and a small modulation (perturbing signal) representing the noise superimposed on it. The optical power $P$ and the carrier density $N$ can be linearized and described as

$$
\begin{aligned}
& P=P_{0}+\frac{1}{2 \pi} \int_{-\infty}^{+\infty} \Delta P(\Omega) e^{j \Omega t} d \Omega \\
& N=N_{0}+\frac{1}{2 \pi} \int_{-\infty}^{+\infty} \Delta N(\Omega) e^{j \Omega t} d \Omega
\end{aligned}
$$

where $P_{0}, N_{0}$ are the time-averages and $\Delta P(\Omega)$ and $\Delta N(\Omega)$ stand for the noise. $\Omega$ is the angular frequency at which the perturbing signal varies. By substituting (3) and (4) into (1) and (2) and considering the component with angular frequency $\Omega$, we get the small signal equations for $\Delta P \equiv \Delta P(\Omega)$ and $\Delta N \equiv \Delta N(\Omega)$ as

$$
\begin{aligned}
\frac{\partial \Delta P}{\partial z} & =j \frac{\Omega}{v_{g}} \Delta P+\left[\Gamma g(N)-\alpha_{\mathrm{int}}\right] \Delta P+\Gamma g^{\prime} \Delta N P_{0}(z) \\
\Delta N & =-\frac{\Gamma g\left(N_{0}\right) \Delta P}{h \nu A} \frac{1}{j \Omega+\frac{1}{\tau_{d}}+\frac{\Gamma g^{\prime} P_{0}}{h \nu A}}
\end{aligned}
$$

where $g^{\prime}$ is the differential gain coefficient.

Equation (6) gives the magnitude of the perturbation to the carrier population, which describes the response of the carrier density due to the amplification of the perturbing signal. It shows that the response has a high frequency rolloff with a 3 - $\mathrm{dB}$ frequency of $1 /\left(2 \pi \tau_{\text {eff }}\right)$ governed by the local effective lifetime $\tau_{\text {eff }}$

$$
\frac{1}{\tau_{\text {eff }}}=\frac{1}{\tau_{d}}+\frac{1}{\tau_{s}}
$$


where $1 / \tau_{s}=\Gamma g^{\prime} P_{0} / A h \nu$ is the contribution of the stimulated emission.

The behavior of the optical power fluctuation in the beam is governed by (5). The last term represents coupling of light from the continuous-wave $(\mathrm{CW})$ component into the time-varying component and acts to compress the gain. This gain compression effect vanishes when the optical power is small. And it is such gain compression that gives rise to a suppression of the optical power fluctuation in the beam, and thus, a noise reduction.

From (5) and (6), we have (with $L$ the length of the SOA)

$$
\begin{aligned}
& \frac{\Delta P(L)}{\Delta P(0)}=e^{j\left(\Omega L / v_{g}\right)} e^{-\alpha_{\mathrm{int}} L} \\
& \cdot \exp \left\{\int_{0}^{L} d z \frac{\Gamma g\left(N_{0}\right)\left(j \Omega+1 / \tau_{d}\right)}{j \Omega+\frac{1}{\tau_{d}}+\frac{\Gamma g^{\prime} P_{0}(z)}{A h \nu}}\right\} .
\end{aligned}
$$

From this, the relative intensity noise (RIN) suppression ratio (electrical) $\eta$ can be derived as in decibel

$$
\begin{aligned}
\eta & =-10 \log \left(\left|\frac{\frac{\Delta P(L)}{P_{0}(L)}}{\frac{\Delta P(0)}{P_{0}(0)}}\right|^{2}\right) \\
& =20(\log e) \int_{0}^{L} d z \frac{\Gamma g\left(N_{0}\right) \frac{\Gamma g^{\prime} P_{0}}{A h \nu}\left(\frac{1}{\tau_{d}}+\frac{\Gamma g^{\prime} P_{0}(z)}{A h \nu}\right)}{\Omega^{2}+\left(\frac{1}{\tau_{d}}+\frac{\Gamma g^{\prime} P_{0}(z)}{A h \nu}\right)^{2}}
\end{aligned}
$$

where

$$
P_{0}(L) / P_{0}(0)=\exp \left[\int_{0}^{L} d z\left(\Gamma g\left(N_{0}\right)-\alpha_{\mathrm{int}}\right)\right] .
$$

Equation (9) shows that $P_{0}, \Gamma g\left(N_{0}\right)$ and $\Gamma g^{\prime}$ have to be as large as possible for getting a high noise reduction. $A$ must be as small as possible. For a given SOA, $P_{0}$ and $\Gamma g\left(N_{0}\right)$ depend on the input power $P_{\text {in }}$ and the injected current $I_{b}$. For a given injected current to the SOA, $P_{0}$ increases with the increase of the input power $P_{\mathrm{in}}$, and $\Gamma g\left(N_{0}\right)$ changes very little when $P_{\text {in }}$ is low. The amount of the noise reduction is, thus, small, but increases with increasing input power. For high input powers, that means, in the case of saturation of the SOA, $P_{0}$ increases very slowly and $\Gamma g\left(N_{0}\right)$ decreases with the increase of the input power. The noise reduction has, thus, a weak dependence on the input power to the saturated SOA. For a given input power, both $P_{0}$ and $\Gamma g\left(N_{0}\right)$ increase rapidly, and thus, the amount of the intensity noise reduction increases significantly by increasing the injected current. Increasing the bias current can also result in an increase in the bandwidth of the noise reduction since the bandwidth increases with the increase of $P_{0}$. So, increasing the bias current of the saturated SOA is the most effective way to get a great reduction of the intensity noise, and thus, a large increase of the signal-to-noise ratio (SNR). These conclusions will be verified by the experimental results given in the following section.

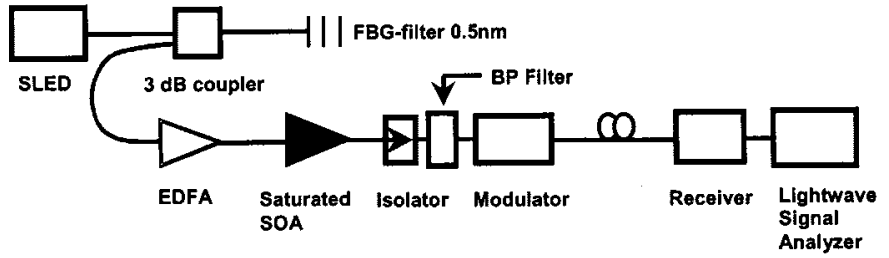

Fig. 1. Experimental setup for intensity noise reduction.

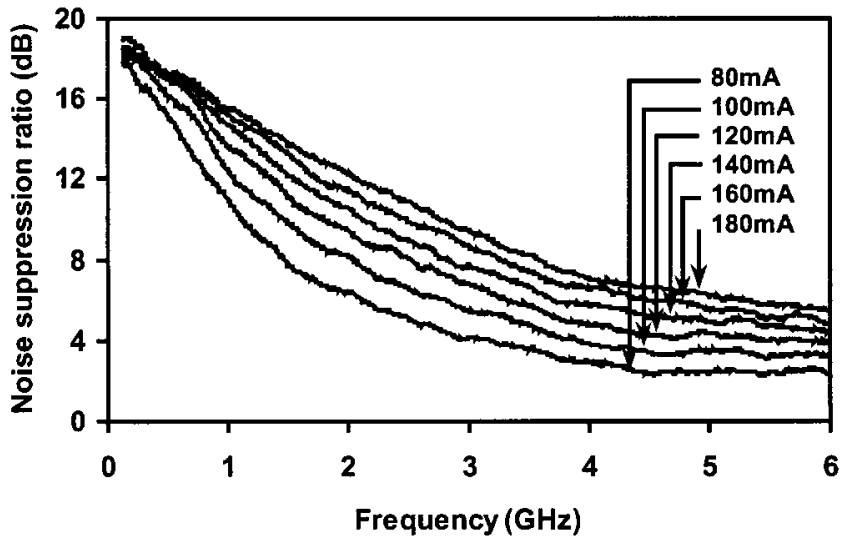

Fig. 2. Measured noise suppression ratios for different bias currents $I_{b}$ for $P_{\mathrm{in}}=4.5 \mathrm{dBm}$.

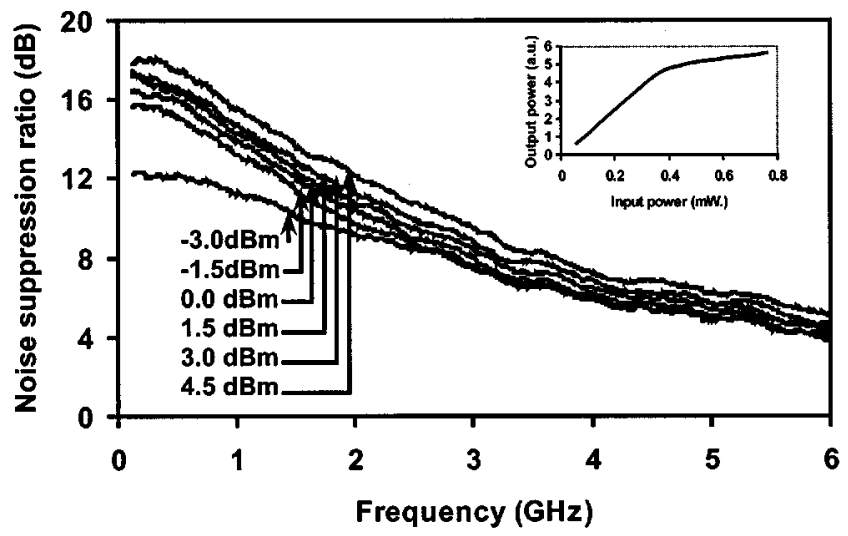

Fig. 3. Measured noise suppression ratios for different input powers $P_{\text {in }}$ for $I_{b}=180 \mathrm{~mA}$. Inset: Saturation characteristic of the SOA.

\section{EXPERIMENTAL RESULTS}

Our experimental setup for the intensity noise reduction using a saturated SOA in the spectrum-sliced WDM system is shown in Fig. 1. The amplified spontaneous emission (ASE) light from a superluminescent LED (SLED) was spectrally sliced using a 0.5-nm bandwidth fiber Bragg grating (FBG) filter with $25 \mathrm{~dB}$ out of channel rejection. The spectrum-sliced incoherent light centered at $1550.8 \mathrm{~nm}$ was amplified using an erbium-doped fiber amplifier (EDFA) and then injected into a gain saturated SOA. The output of the SOA was modulated using a $\mathrm{LiNbO}_{3}$ external modulator. To remove the ASE from the amplifiers and the lasing-mode from the SOA, a 1.2-nm bandwidth filter was used before the modulator. The saturation characteristic of the SOA (SOA Module-GC187, Alcatel) used in the experiments is shown as an inset in Fig. 3. 


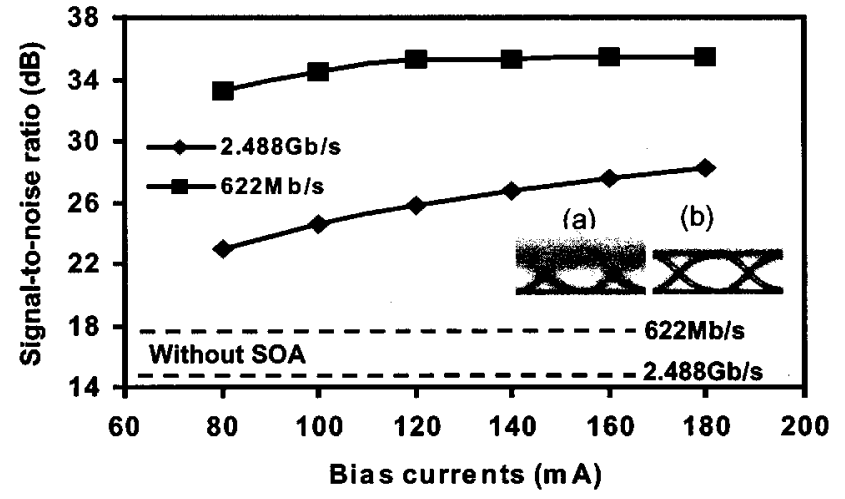

Fig. 4. Signal-to-noise ratios versus bias currents for two different bit rates at back-to-back operation. $P_{\text {in }}=4.5 \mathrm{dBm}$. Inset: Eye diagrams for a data bitrate of $2.488 \mathrm{~Gb} / \mathrm{s}$. (a) Without SOA. (b) With SOA.

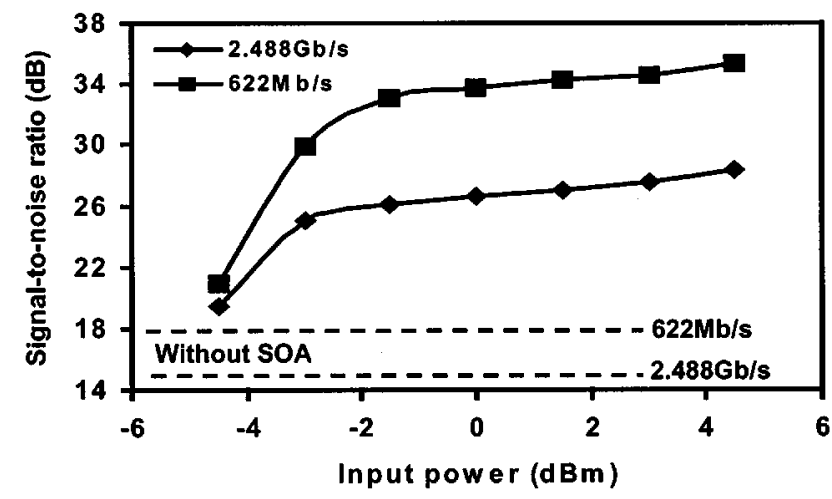

Fig. 5. Signal-to-noise ratios versus input powers for two different bit rates at back-to-back operation. $I_{b}=180 \mathrm{~mA}$.

In the experiments, we measured the RIN spectra of the spectrum-sliced light with and without the saturated SOA, respectively. The RIN level of the optical slice was around $-110 \mathrm{~dB} / \mathrm{Hz}$ without the SOA. With the SOA, it was significantly suppressed at low frequencies over a bandwidth of several GHz. Figs. 2 and 3 show the noise suppression ratios as a function of frequency measured for different bias currents and different input powers to the SOA, respectively. Clearly, the bandwidth of the noise reduction increases by increasing the bias current of the SOA, but not efficiently by increasing the input power. The amount of the noise reduction depends on the bias current and the input power. The noise reduction can increase by increasing both of them, but the former is a more effective way. The experimental results are consistent with the analytical predictions. For very low frequencies and large optical power, the noise suppression ratio can be up to $18 \mathrm{~dB}$. This is in relatively good agreement with the $20 \mathrm{~dB}$ estimated from (9), where a measured fiber-to-fiber amplification of $7.5 \mathrm{~dB}$, a chip-to-fiber coupling loss of $3 \mathrm{~dB}$, and an internal loss of $25 \mathrm{~cm}^{-1}$ of the SOA were used and $\tau_{d}$ and $\tau_{s}$ were assumed to be equal.

Figs. 4 and 5 show the intensity-noise-limited SNR's with and without SOA at bitrates of $622 \mathrm{Mb} / \mathrm{s}$ and $2.488 \mathrm{~Gb} / \mathrm{s}$ for different bias currents and input powers to the SOA, respectively. The SNR can be enhanced significantly by increasing the bias current of the saturated SOA for high bitrate, e.g., $2.488 \mathrm{~Gb} / \mathrm{s}$. But at a low bitrate, e.g., $622 \mathrm{Mb} / \mathrm{s}$, the enhancement in the SNR is not sensitive to the bias current. This is because the receiver bandwidth is very small for this bitrate, and thus, the increase of the bandwidth of the noise reduction gives less contribution to the SNR enhancement. For a given bias current, the improvement of the SNR is very small when the input power is very low and the SOA is unsaturated. For large input powers, the SOA is saturated, and thus, a large improvement of the SNR is obtained. The improvement of the SNR is insensitive to the input power to the saturated SOA. This can be explained by the fact that both the bandwidth and the amount of the noise reduction are insensitive to the input power.

With a high bias current of $180 \mathrm{~mA}$, an improvement on the SNR of $13.5 \mathrm{~dB}$ at a bitrate of $2.488 \mathrm{~Gb} / \mathrm{s}$ and $17.5 \mathrm{~dB}$ at $622 \mathrm{Mb} / \mathrm{s}$ have been obtained experimentally in a single stage SOA for an input power of $4.5 \mathrm{dBm}$. Eye diagrams for $2.488 \mathrm{~Gb} / \mathrm{s}\left(2^{23}-1\right.$ PRBS) back-to-back operation with and without the SOA are shown in Fig. 4 as an inset. Clearly, the intensity noise is suppressed dramatically. The achievable bitrate using a SOA for noise reduction depends on both intrinsic parameters (e.g., $\tau_{d}, \Gamma g^{\prime}, A$ ) and operation conditions (e.g., bias current, input power ) of the SOA and can be estimated from the measured RIN spectra. For a high bias current of $180 \mathrm{~mA}$ and an input power of $4.5 \mathrm{dBm}$, a bitrate of $5 \mathrm{~Gb} / \mathrm{s}$ with a SNR of $20 \mathrm{~dB}$ can be achieved with the SOA used in our experiments in a single stage. If cascaded saturated SOAs are used, the achievable bitrate can be increased.

\section{CONCLUSION}

We have presented experimental results on the intensity noise suppression with a gain-saturated SOA in spectrum-sliced WDM systems. The influence of the input power level and the bias current of the SOA on the noise reduction and its bandwidth is studied theoretically and experimentally. It was found that the most effective way for getting a high noise suppression ratio and a large bandwidth is to increase the bias current of the saturated SOA. For the optimum operation of the SOA, an increase of $13.5 \mathrm{~dB}$ in the intensity-noise-limited signal-to-noise ratio (SNR) for a bitrate of $2.488 \mathrm{~Gb} / \mathrm{s}$ and of $17.5 \mathrm{~dB}$ for a bitrate of $622 \mathrm{Mb} / \mathrm{s}$ are obtained experimentally. These results provide useful information for the design and performance improvement of the spectrum-sliced WDM networks.

\section{REFERENCES}

[1] G. J. Pendock and D. D. Sampson, "Transmission performance of high bit rate spectrum-sliced WDM systems," J. Lightwave Technol., vol. 14, pp. 2141-2148, Oct. 1996.

[2] F. Koyama, "High power superluminescent diodes for multi-wavelength light source," in IEEE LEOS Annu. Meeting, 1997, Paper TuY2, pp. 333-334.

[3] S. J. Kim, J. H. Han, J. S. Lee, and C. S. Park, "Suppression of intensity noise in $10 \mathrm{Gbit} / \mathrm{s}$ spectrum-sliced incoherent light channel using gainsaturated semiconductor optical amplifiers," Electron. Lett., vol. 35, pp. 1000-1001, 1999.

[4] T. Yamatoya, F. Koyama, and K. Iga, "Noise suppression and intensity modulation using gain-saturated semiconductor optical amplifier," in Tech. Dig. Integrated Photonics Research 2000, Canada, 2000, pp. 72-74.

[5] M. E. Bray and M. J. O’Mahony, "Cascading gain-saturation semiconductor laser-amplifier wavelength translators," Proc. Inst. Elect. Eng.Optoelectron., vol. 143, no. 1, pp. 1-6, 1996. 